\title{
Evolutionary Relationships among Bacterial Carbamoyltransferases
}

\author{
By CATHERINE TRICOT, ${ }^{1}$ JEAN-LOUIS DE COEN,${ }^{2}$ PATRICIA \\ MOMIN, ${ }^{1}$ PAUL FALMAGNE ${ }^{3}$ AND VICTOR STALON ${ }^{1 *}$ \\ ${ }^{1}$ Laboratoire de Microbiologie, Faculté des Sciences, Université Libre de Bruxelles \& Institut de \\ Recherches du CERIA, I avenue E. Gryson, B-1070 Brussels, Belgium \\ ${ }^{2}$ Laboratoire de Chimie Générale I, Faculté des Sciences, Université Libre de Bruxelles, \\ 50 avenue F. D. Roosevelt, B-1050 Brussels, Belgium \\ ${ }^{3}$ Laboratoire de Chimie Biologique, Faculté des Sciences, Université de l'Etat à Mons, \\ 21 avenue Maistriau, B-7000 Mons, Belgium
}

(Received 7 February 1989; revised 17 May 1989; accepted 6 June 1989)

\begin{abstract}
An immunological approach was used for the study of ornithine carbamoyltransferase (OTCase) evolution in bacteria. Antisera were prepared against the anabolic and catabolic OTCases of Pseudomonas aeruginosa and Aeromonas formicans as well as against OTCase and putrescine carbamoyltransferases from Streptococcus faecalis; these antisera were then tested against the unpurified OTCases, either anabolic or catabolic, of 34 bacterial strains. Extensive crossreactions were observed between the antisera to catabolic OTCases from $P$. aeruginosa, $A$. formicans and $S$. faecalis and the catabolic enzymes from other species or genera. These antisera cross-reacted also with the anabolic OTCases of strains of the Enterobacteriaceae but not with the anabolic OTCases of the same species or of other species or genera. The cross-reaction measured between the antisera against $P$. aeruginosa anabolic OTCase and the anabolic OTCases of other Pseudomonas were largely in agreement with the phylogenic subdivision of Pseudomonas proposed by N. J. Palleroni. The correlation was also significantly higher with the anabolic enzyme of an archaeobacterium, Methanobacterium thermoaceticum, than with the catabolic or anabolic OTCases from other genera in the eubacterial line. The antiserum raised against $A$. formicans anabolic OTCase was quite specific for its antigen and appeared to be raised against the heaviest of the various oligomeric structures of the enzyme.
\end{abstract}

\section{INTRODUCTION}

Anabolic ornithine carbamoyltransferase (OTCase; EC 2.1.3.3) functions in the biosynthesis of arginine and catalyses the formation of citrulline and phosphate from ornithine and carbamoylphosphate. The anabolic OTCase from a variety of organisms occurs as a trimeric molecule with a subunit molecular mass ranging from 35 to $40 \mathrm{kDa}$. This trimeric structure also exists in other enzymes involved in carbamoylphosphate metabolism, namely in aspartate carbamoyltransferase (ATCase; EC 2.1.3.2) and in putrescine carbamoyltransferase (PTCase; EC 2.1.3.6) (Vickers, 1981; Cunin et al., 1986).

Catabolic OTCase is part of the catabolic arginine deiminase pathway and catalyses the formation of carbamoylphosphate and ornithine from citrulline and phosphate. Catabolic OTCases are usually large proteins composed of three, six, eight, nine or more identical subunits (Legrain et al., 1977; Marshall \& Cohen, 1972; Baur et al., 1987).

Arginine prototrophic organisms that possess the arginine deiminase pathway have two OTCases, one anabolic and the other catabolic. In Pseudomonas putida and Pseudomonas aeruginosa, both enzymes function unidirectionally. The Pseudomonas catabolic enzymes display intense allosteric behaviour which probably results from their large oligomeric structure,

\footnotetext{
Abbreviations: ATCase, aspartate carbamoyltransferase; OTCase, ornithine carbamoyltransferase; PTCase, putrescine carbamoyltransferase.
} 
octameric in $P$. putida OTCase, nonameric or dodecameric in the $P$. aeruginosa enzyme (Baur et $a l .$, 1987). In this context it is not clear whether trimeric and multimeric OTCases are similar proteins resulting from convergence, or homologous proteins resulting from the differentiation and specialization of an ancestral carbamoyltransferase.

To answer this question we have determined the amino acid sequence of the two $P$. aeruginosa OTCases (Baur et al., 1987; Itoh et al., 1988) and compared them with the known primary structure of other bacterial OTCases and ATCases. All these enzymes bear substantial sequence similarities which indicate a close evolutionary relationship.

More information concerning the structural similarities between the carbamoyltransferases was obtained by exploring the immunological relationships of the enzymes from various bacteria. Previous immunological studies (Falmagne et al., 1985) have shown that the catabolic OTCase from $P$. putida shares common antigenic determinants with other catabolic OTCases and also with the anabolic OTCase from Enterobacteriaceae. Using specific antisera directed against the $P$. putida catabolic enzyme or the $E$. coli anabolic OTCase we have observed no heterologous cross-reactions with either the $P$. putida anabolic OTCase or the anabolic OTCases from representatives of other bacterial genera (Falmagne et al., 1985). In the present work we have used antisera against the anabolic and catabolic enzymes from $P$. aeruginosa and Aeromonas formicans as well as against OTCase and PTCase from Streptococcus faecalis. These enzymes were selected for three reasons. Firstly, the primary structure of both the anabolic and catabolic OTCases from $P$. aeruginosa PAO has recently been determined; secondly, the enzymes from Aeromonas have been shown to exist in various active aggregates (Legrain et al., 1977) which could have different immunological properties; thirdly, PTCase displays OTCase activity and, for this reason, was included in the immunochemical comparison with the OTCases. Finally, whenever possible, $\mathrm{NH}_{2}$-terminal sequences were determined for purified enzymes in order to complete their structural comparison.

\section{METHODS}

Strains. The strains used and their origins are listed in Table 1. Methanobacterium thermoautotrophicum (DMS 2133) was obtained from T. Leisinger, Zürich, Switzerland.

Media and growth conditions. Conditions for bacterial growth and cell-free extract preparation have been described previously (Falmagne et al., 1985). Lactic bacteria were grown in the presence of 20 mM-glucose, yeast extract and tryptone, as described by Wargnies et al. (1979). Arginine ( $50 \mathrm{~mm}$ ) was used as an added energy source for the production of the catabolic OTCase, whereas $25 \mathrm{~mm}$-agmatine was added for the production of PTCase.

Enzyme assays. The anabolic OTCases were routinely assayed in $50 \mathrm{mM}-\mathrm{EDTA} / \mathrm{NaOH}$ buffer, $\mathrm{pH} 8.5$, containing $10 \mathrm{~mm}$ each of carbamoylphosphate and ornithine. The anabolic OTCase of Aeromonas was assayed in the presence of $50 \mathrm{~mm}$-ornithine. The catabolic OTCases from Pseudomonas were assayed as described by Falmagne et al. (1985); the catabolic OTCase and PTCase from lactic bacteria were assayed as in Wargnies et al. (1979); the anabolic and catabolic OTCases from Bacillus licheniformis were assayed as described by Broman $e$ t al. (1978). The OTCase from Methanobacterium thermoautotrophicum was assayed according to Meile \& Leisinger (1984).

The detailed preparation of the purified transcarbamylases used in this study is described in Baur et al. (1987) for the $P$. aeruginosa PAO catabolic OTCase, in Itoh $e$ t al. (1988) for the $P$. aeruginosa anabolic OTCase, in Wargnies et al. (1979) for the OTCase and PTCase from S. faecalis, in Falmagne et al. (1985) for the E. coli argF and argI products and in Legrain et al. (1977) for the catabolic OTCase from Bacillus and Aeromonas. The catabolic OTCase of $P$. putida was purified according to Halleux et al. (1972). The catalytic subunit of $E$. coli K12 ATCase was a gift from G. Hervé (Gif-sur-Yvette, France).

Purification of the anabolic OTCase from A. formicans. A. formicans NCIB 9232 was grown aerobically at $30^{\circ} \mathrm{C}$ on minimal medium 154 (Stalon et al., 1972) supplemented with glucose and ammonia (Stalon \& Mercenier, 1984) in a 151 fermenter. Cells were collected by centrifugation at the end of the exponential phase $\left(8 \times 10^{8} \mathrm{cells}^{-1}\right)$ and sonicated (Legrain et al., 1977) in the presence of $200 \mathrm{ml}$ EDTA/NaOH buffer $(25 \mathrm{mM}, \mathrm{pH} 6.8)$ containing $100 \mathrm{mM}$ each of potassium phosphate and ornithine, and $3 \mathrm{~mm}-2$-mercaptoethanol. After centrifugation at $20000 \mathrm{~g}$ for $20 \mathrm{~min}$ the crude extract was heated at $60^{\circ} \mathrm{C}$ for $15 \mathrm{~min}$. The coagulated solids were discarded by centrifugation. The supernatant was fractionated by ammonium sulphate precipitation and the $30-60 \%$ saturation fraction was collected. This fraction was dialysed against $25 \mathrm{~mm}$-EDTA/NaOH buffer, $\mathrm{pH} 6.8$, and applied to a DEAESepharose CL6B column $(5 \times 40 \mathrm{~cm})$ equilibrated with the same buffer. OTCase was eluted with a linear gradient of potassium phosphate $(50-400 \mathrm{~mm} ; 800 \mathrm{ml})$, concentrated by ultrafiltration with a Diaflow PM10 membrane 
Table 1. Bacterial strains used in this investigation

\begin{tabular}{|c|c|c|c|}
\hline Species & $\begin{array}{c}\text { Strain or } \\
\text { source }\end{array}$ & Species & $\begin{array}{c}\text { Strain or } \\
\text { source }\end{array}$ \\
\hline Pseudomonas aeruginosa & PAO & Proteus vulgaris & IRC 9623 \\
\hline Pseudomonas putida & IRC 204 & Escherichia coli $\mathrm{C} 600 \mathrm{OTC}^{-} \Delta(\mathrm{pMC} 20)$ & This laboratory \\
\hline Pseudomonas putida & ATCC 12633 & Escherichia coli $\mathrm{C} 600$ OTC $^{-} \Delta(\mathrm{pA} 11)$ & This laboratory \\
\hline Pseudomonas fuorescens & ATCC 13525 & Aeromonas formicans & NCIB 9232 \\
\hline Pseudomonas aureofaciens & ATCC 13985 & Streptococcus faecalis & ATCC 11700 \\
\hline Pseudomonas mendocina & NCIB 10541 & Streptococcus faecalis & IRC \\
\hline Pseudomonas cepacia & NCTC 10743 & Streptococcus faecalis & ATCC 14508 \\
\hline Pseudomonas caryophilli & ATCC 25418 & us faecium & 9790 \\
\hline Pseudomonas syringae & ATCC 19310 & cillus brevis & IP 7135 \\
\hline Pseudomonas stutzeri & ATCC 11607 & lus fermentum & АTCC 9338 \\
\hline Pseudomonas pseudoalcaligenes & ATCC 17440 & Streptococcus lactis & NCIB 8586 \\
\hline Pseudomonas indigofera & ATCC 19706 & Staphylococcus aureus Cowan & ATCC12598 \\
\hline Pseudomonas maltophilia & ATCC 13637 & Bacillus licheniformis & ATCC 14580 \\
\hline Pseudomonas acidovorans & ATCC 17439 & Bacillus subtilis & Marburg C4 \\
\hline Pseudomonas testosteroni & ATCC 11996 & Corynebacterium glutamicum & ATCC 21659 \\
\hline Salmonella typhi & ATCC 6539 & Brevibacterium fluvum & ATCC 21493 \\
\hline Klebsiella aerogenes & MK53* & Agrobacterium tumefaciens & R 10 \\
\hline
\end{tabular}

* Received from B. Magasanik. Massachusetts Institute of Technology, Boston, MA, USA.

(Amicon), and dialysed against $25 \mathrm{mM}-\mathrm{EDTA} / \mathrm{NaOH}$ buffer, $\mathrm{pH} 8 \cdot 0$, supplemented with 50 mM-ornithine and $3 \mathrm{~mm}$-2-mercaptoethanol. A G-200 Sephadex column $(2.5 \times 40 \mathrm{~cm})$ equilibrated with the same buffer was loaded with the OTCase fraction. The fractions containing the highest activities were pooled, concentrated and dialysed against $25 \mathrm{mM}$-EDTA/ $\mathrm{NaOH}$ buffer, pH 6.0, containing $50 \mathrm{~mm}$-potassium phosphate and $100 \mathrm{mM}-\mathrm{KCl}$ and then rechromatographed on a G-200 column $(2.5 \times 40 \mathrm{~cm})$ equilibrated with the same buffer. The fractions containing the highest activities were again pooled, concentrated and dialysed against $25 \mathrm{mM}$-potassium phosphate buffer, $\mathrm{pH} 7.0$, and stored at $-20^{\circ} \mathrm{C}$.

Thermostability. Tris $/ \mathrm{HCl}$ buffer $(50 \mathrm{~mm} ; 1.9 \mathrm{ml} ; \mathrm{pH} 8.0)$ containing the desired effector was heated at $60^{\circ} \mathrm{C}$ for $5 \mathrm{~min}$, then $0.1 \mathrm{ml}$ of the enzyme ( $200 \mathrm{units}$ ) was added to the test-tube and rapidly mixed. At intervals, $50 \mu \mathrm{l}$ samples of the solution were removed and rapidly chilled in a test-tube containing the reaction mixture (minus carbamoylphosphate) for the OTCase assay. After $3 \mathrm{~min}$ the reaction was started at $30^{\circ} \mathrm{C}$ by the addition of carbamoylphosphate.

Molecular sieving: determination of the molecular mass of the Aeromonas anabolic OTCase. A $2.5 \times 40 \mathrm{~cm}$ column was packed full with swollen Sephadex G-200. To assure constant bed-height and equilibration with eluent, the column was washed for $36 \mathrm{~h}$ with eluting buffer containing $3 \mathrm{~mm}-2$-mercaptoethanol in each case. A flow rate of $12 \mathrm{ml} \mathrm{h}^{-1}$ was maintained during the elution process. Complete elution of the OTCase activity was achieved within $8 \mathrm{~h}$. Before each run, the native enzyme (2000 units) was dialysed extensively for $24 \mathrm{~h}$ against $3 \times 11$ changes of buffer (see Results).

Electrophoresis. SDS-PAGE and the cross-linking of the subunits of the native enzyme were done as described previously (Legrain \& Stalon, 1976).

Preparation and analysis of antisera. Purified carbamoyltransferases were used to immunize rabbits. Material for injections was emulsified in an equal volume of Freund's complete adjuvant (total volume $2.0 \mathrm{ml}$ ). For each protein, two animals received first multiple intradermal and one intramuscular injections. Three subsequent injections of enzyme were administered at 3-week-intervals. The total amount of enzyme given to each rabbit was $0.5 \mathrm{mg}$. Each animal was bled on several occasions to test for antisera. Antisera were titrated against the homologous antigen. Those having the highest titre were pooled and stored at $-80^{\circ} \mathrm{C}$. Antisera used for precipitation tests were heated for $30 \mathrm{~min}$ at $56^{\circ} \mathrm{C}$ and coagulated materials were discarded by centrifugation.

Quantitative immunoprecipitation. Quantitative measurements of cross-reactivity were made by incubating various dilutions of antiserum ranging from $10 \mathrm{nl}$ to $100 \mu \mathrm{l}$ of antiserum with a fixed amount of enzyme activity (10 units). A given sample of antiserum was assayed in duplicate or triplicate. The immunoprecipitated antibodies linked to staphylococcal protein A were removed by centrifugation as described by Falmagne $e t$ al. (1985). Tubes with non-immume serum were always included to correct for nonspecific precipitation. With each antiserum, it was checked that similar levels of antiserum were needed to precipitate the specific purified antigen and a cell-free extract preparation. With the exception of $A$. formicans anabolic OTCase (see Results), less than $10 \%$ variation was observed with purified or crude cell extract preparation. In each case, a check was made to ensure than no inhibition of enzyme activity occurred in the range of antibodies used in this study, even when the highest amount 
of antiserum was incubated with its antigen. The titre is defined as the antiserum concentration at which $75 \%$ of the antigen activity was precipitated. The index of dissimilarity (ID) refers to the ratio of antiserum concentration needed to precipitate $75 \%$ of the heterologous and homologous activities. Preparation of antisera, amino acid analysis and $\mathrm{NH}_{2}$-terminal amino acid sequences are described in detail in a previous publication (Falmagne $e t$ al., 1985).

\section{RESULTS}

\section{Anabolic OTCase of A. formicans}

Purification. A. formicans anabolic OTCase was purified from a crude extract of the wild-type strain which had a specific activity of 110 units $^{-1}(\mathrm{mg} \text { protein })^{-1}$. After 1600 -fold purification, as described under Methods, $5 \mathrm{mg}$ of an electrophoretically $95 \%$ homogeneous preparation, specific activity 175000 units $^{-1}$ (mg protein $)^{-1}$, was obtained with a yield of $15 \%$. SDS-PAGE gave a single band corresponding to a subunit of molecular mass of $36 \mathrm{kDa}$. The results of crosslinking experiments followed by SDS-PAGE revealed five major bands; when the migration of these bands was plotted versus the molecular mass, the covalently cross-linked species produced by amidination appeared to be multiples of the $36 \mathrm{kDa}$ protomer. The five protein species corresponded respectively to the monomer, the dimer, the trimer, the hexamer and the nonamer of the subunit. Increasing the amidination time increased the amount of the higher molecular mass species and decreased that of the low molecular mass species. This indicates that the higher molecular mass species may well result from an association of trimeric species. With an extended amidination time a minor additional band of higher molecular mass than the nonamer could be observed, suggesting a small amount of intermolecular cross-linking.

Molecular sieving : molecular mass determination. The molecular mass of the native enzyme was estimated by molecular sieving in the presence of several eluents. Representative results are given in Fig. 1. Depending on the conditions used, two molecular forms of the enzyme were obtained. Under acidic conditions ( $\mathrm{pH} \mathrm{6.0)}$ in the presence of either $25 \mathrm{~mm}$-citrate/ $\mathrm{NaOH}$, $25 \mathrm{~mm}$-EDTA/ $\mathrm{NaOH}$ or $25 \mathrm{~mm}$-potassium phosphate, the prominent activity eluted at a molecular mass of $360 \mathrm{kDa}$, corresponding probably to a nonameric species. In contrast, basic $\mathrm{pH}$ (8.5) increased the amount of a trimeric species with a molecular mass of $120 \mathrm{kDa}$. Dissociation into trimers could also be promoted by ornithine and phosphate, or by ethylene glycol and other non-aqueous solvents. Enzyme dissociation was prevented by salt $(100 \mathrm{~mm}-\mathrm{KCl})$. In all cases, the molecular mass of the enzyme was unaffected by the absence or presence of thiol agents (10 mM-2-mercaptoethanol), thus indicating that the bonds between the subunit are entirely noncovalent. The same pattern of enzyme distribution was observed with either crude or purified preparations. The variation of molecular mass of the native enzyme with $\mathrm{KCl}$, ethylene glycol and $\mathrm{pH}$ suggests that hydrophobic bonds are involved in the quaternary structure of this enzyme.

Thermostability of the enzyme. When purified enzyme was incubated in $50 \mathrm{~mm}-\mathrm{Tris} / \mathrm{HCl}$ buffer, $\mathrm{pH} 8 \cdot 8$, at $60^{\circ} \mathrm{C}$, the half-life of the enzyme was about $10 \mathrm{~min}$. However, when $10 \%(\mathrm{v} / \mathrm{v})$ ethylene glycol or $10 \mathrm{~mm}$-ornithine was added to the buffer, the enzyme was more rapidly inactivated (half-life 2-3 min). The presence of $100 \mathrm{mM}-\mathrm{KCl}$ prolonged the half-life to about $2 \mathrm{~h}$.

These results may indicate that the different conformational states of the enzyme have different thermostabilities and suggest that the most dissociated active form of the enzyme is the most labile. This is strengthened by the fact that the molecular mass species of $120 \mathrm{kDa}$ obtained after gel filtration was completely destroyed within $1 \mathrm{~min}$ exposure to $60^{\circ} \mathrm{C}$, while the molecular mass species of $360 \mathrm{kDa}$ had a half-life of about $15 \mathrm{~min}$ under the same conditions.

\section{Specificity of the antiserum to OTCases of $A$. formicans and $P$. aeruginosa}

Confirming our previous results, antisera raised against the catabolic OTCase of either $A$. formicans or $P$. aeruginosa cross-reacted with the other catabolic OTCases (Table 2). The corresponding enzymes of Gram-positive bacteria showed lower activity with the two antisera. In addition, cross-reactivity was also observed with some anabolic enzymes of Enterobacteriaceae. However, strong differences in the cross-reactivities of the two antisera were observed 


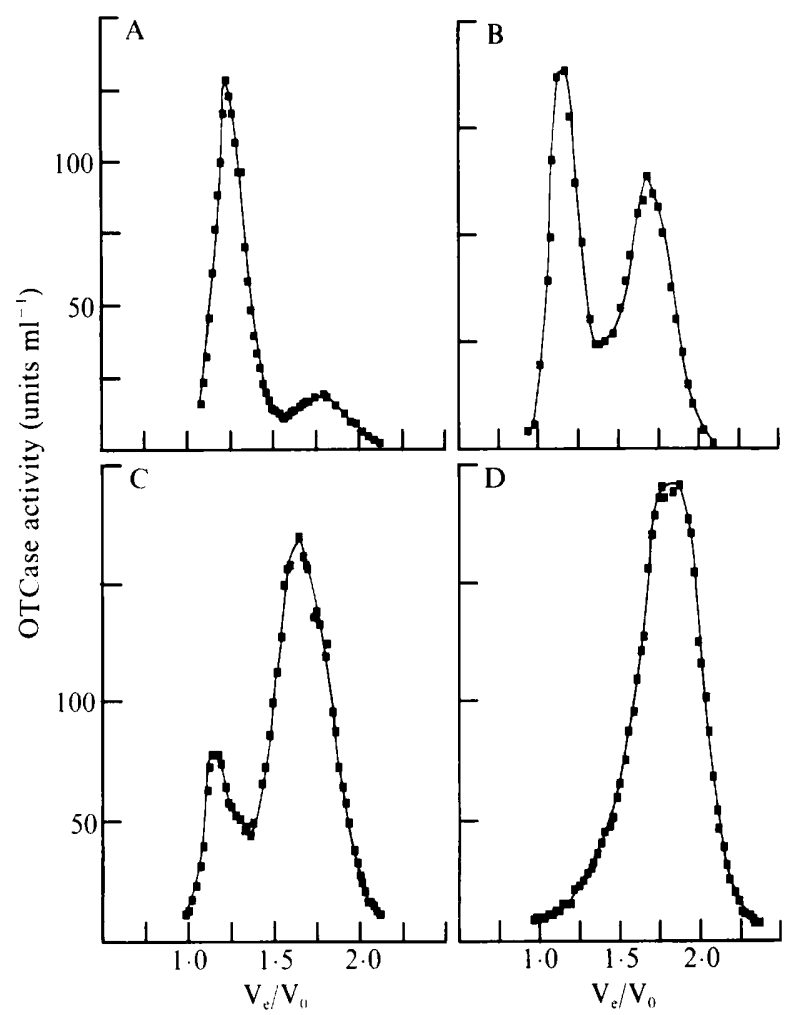

Fig. 1. Distribution of $A$. formicans anabolic OTCase activity after gel filtration on a Sephadex G200 column $(2.5 \times 40 \mathrm{~cm})$. $V_{e}$, elution volume; $V_{0}$, void volume. Elution buffers used were: $A$, potassium phosphate (50 mM, pH 6.0); B, potassium phosphate $(50 \mathrm{~mm}, \mathrm{pH} 8.0$ ) supplemented with $50 \mathrm{~mm}$ ornithine; C, EDTA/NaOH (20 mM, pH 8.5) supplemented with 50 mM-ornithine; D, EDTA/NaOH $(20 \mathrm{mM}, \mathrm{pH} 8.5)$ supplemented with $50 \mathrm{~mm}$-ornithine and $25 \%(\mathrm{v} / \mathrm{v})$ ethylene glycol.

with $\arg F$ and $\arg I$ products of $E$. coli $\mathrm{K} 12$ (Table 2). These isoenzymes show an overall identity of $86 \%$ at the amino acid level (Van Vliet et al., 1984), while they present a $57.1 \%$ and $55.7 \%$ identity respectively with the $P$. aeruginosa catabolic OTCase (Baur et al., 1987). This strong discrepancy between the cross-reactivity of the two $E$. coli isoenzymes with the $P$. aeruginosa or A. formicans catabolic OTCase (Table 2) could conceivably be due to only a few key antigenic sites on the enzyme molecules. Consequently, any alteration in those sites could greatly exaggerate the dissimilarity established by immunological tests. A simple method for predicting antigenic protein determinants from amino acid sequence was described by Hopp \& Woods (1985). It involves the calculation of local hydrophilicity values using the six amino acids surrounding each position in the sequence, and plotting hydrophilicity against position. Hopp and Woods have shown, for different proteins studied previously, that the major hydrophilicity peaks correspond to antigenic determinants. The rules of Hopp and Woods, when applied to the amino acid sequences of $P$. aeruginos $a$ catabolic OTCases and of the $E$. coli anabolic isoenzymes, showed that a hydrophilicity peak present in the profile of $P$. aeruginosa OTCase and of $E$. coli $\arg F$ isoenzyme is absent in the $E$. coli $\arg I$ OTCase. This hydrophilicity peak is located at residues 201-210 with a magnitude of 1.3 in the catabolic OTCase and 1.2 in the $\arg F$ isoenzyme, whereas the same region appears to be fairly hydrophobic in the $\arg I$ sequence. However, the prediction rules of Hopp and Woods do not take into account the fact that hydrophobic and aromatic residues are often implicated in epitopes. On the other hand, regions corresponding to the amino- or carboxy-terminus often elicit antibody formation (Westhof et al., 1984). In this context, it is noteworthy that both the catabolic OTCase and the $E$. coli argF OTCase bear 
Table 2. Indices of dissimilarity for OTCases with the catabolic OTCases from P. aeruginosa and $A$. formicans as reference points

\begin{abstract}
Values are representative of two experiments; variations are less than $5 \%$. The titre (nl antiserum required to precipitate $75 \%$ of 10 units of antigen activity) was $21 \mathrm{nl}$ and $43 \mathrm{nl}$ for the catabolic OTCases from $A$. formicans and $P$. aeruginosa respectively. The numbers in parentheses indicate the true dissimilarity index which is defined as the ratio of antiserum concentration needed to precipitate $1 \mathrm{pmol}$ of heterologous and homologous antigen. Previously published data for the turnover number of catabolic OTCases for $P$. putida (Halleux et al., 1972), P. aeruginosa (Baur et al., 1987), Streptococcus faecalis (Wargnies et al., 1979) were used. In the calculation, values for $E$. coli K12 OTCase isoenzymes were similar, as previously found for $E$. coli W (Legrain \& Stalon, 1976).
\end{abstract}

\begin{tabular}{|c|c|c|c|}
\hline \multirow[b]{2}{*}{ Enzyme source } & \multirow[b]{2}{*}{$\begin{array}{l}\text { Enzyme } \\
\text { function }\end{array}$} & \multicolumn{2}{|c|}{ Index of dissimilarity } \\
\hline & & $\begin{array}{l}\text { Anti- } \\
\text { A. formicans } \\
\text { OTCase }\end{array}$ & $\begin{array}{l}\text { Anti- } \\
P . \text { aeruginosa } \\
\text { OTCase }\end{array}$ \\
\hline Aeromonas formicans NCIB 9232 & $\mathrm{C}$ & 1 & $1.6(10 \cdot 4)$ \\
\hline Pseudomonas aeruginosa PAO & $\mathrm{C}$ & $192(42)$ & 1 \\
\hline Pseudomonas putida IRC 204 & $\mathrm{C}$ & $941(128)$ & $3 \cdot 3(2 \cdot 3)$ \\
\hline Pseudomonas putida ATCC 12633 & $\mathrm{C}$ & NT & 3.4 \\
\hline Pseudomonas fluorescens ATCC 13525 & $\mathrm{C}$ & 1322 & 6.0 \\
\hline Pseudomonas mendocina NCIB 10541 & $\mathrm{C}$ & 340 & $1 \cdot 2$ \\
\hline Proteus vulgaris IRC 9623 & A & 394 & $3 \cdot 5$ \\
\hline Escherichia coli $\mathrm{K} 12$ arg $F$ product & A & $71(25)$ & $3 \cdot 7(8 \cdot 2)$ \\
\hline Escherichia coli $\mathrm{K} 12$ argI product & A & $780(279)$ & NC \\
\hline Klebsiella aerogenes MK53 & A & 617 & NT \\
\hline Salmonella typhi ATCC 6539 & $\mathbf{A}$ & $\mathrm{NC}$ & $3 \cdot 7$ \\
\hline Bacillus licheniformis ATCC 14580 & $\mathrm{C}$ & + & 64 \\
\hline Streptococcus faecalis ATCC 11700 & $\mathrm{C}$ & + & $181(801)$ \\
\hline
\end{tabular}

Abbreviations: NC, no cross-reaction; NT, not tested; A, anabolic OTCase; C, catabolic OTCase; + , positive cross-reaction at the highest level of antiserum concentration $(100 \mu \mathrm{l})$.

anionic residues in the carboxy-terminal region while a lysine is present at the same position in the $E$. coli argI product. Finally, antibodies could be directed against topographic determinants involving residues far apart in the amino acid sequence, but brought together on the surface of the native molecule. The residues where the $E$. coli argI product differs from both the $\arg F$ product and the catabolic OTCase of Pseudomonas are Gln41, Gly122, the tripeptide Leu-AlaAsp at position 139-141, the lysine residues at positions 190, 224 and 242, Ala200, Glu207 and Glu242. Anionic residues appear at position 196-197 in both the catabolic enzyme from Pseudomonas PAO and E. coli $\arg F$ isoenzyme, whereas two alanines are present in the corresponding $\operatorname{argI}$ sequence. The precise delineation of antigenic domains will require a complete structure determination by X-ray diffraction analysis, and this is under way for the $P$. aeruginosa catabolic OTCase.

Antiserum to $A$. formicans and to $P$. aeruginosa catabolic OTCases do not cross-react with the anabolic enzymes of other bacteria including their own genera. The hydrophilicity profile of the $P$. aeruginosa anabolic enzyme also showed peaks of hydrophilicity quite different from those of either the catabolic enzyme of the same organism or of the $E$. coli $\arg F$ and $\arg I$ anabolic isoenzymes.

\title{
Specificity of the antiserum to A. formicans anabolic OTCase
}

Quite different levels of antisera were needed to precipitate the crude and the purified anabolic OTCase. However, when the preparations were dialysed in the presence of various effectors, the same levels of antisera were required to precipitate both enzymes. The enzyme dialysed in $50 \mathrm{~mm}$-potassium phosphate, $\mathrm{pH} 7.0$, with $100 \mathrm{mM}-\mathrm{KCl}$ required the lowest level of antiserum, $20 \mathrm{nl}$, while in the presence of $10 \%$ ethylene glycol five times more antiserum was required to precipitate the enzyme dialysed against the same buffer adjusted to $\mathrm{pH} 8.0$. In addition, when ornithine was included in the dialysis buffer, only partial precipitation $(60 \%)$ 
Table 3. Indices of dissimilarity for anabolic OTCases with P. aeruginosa anabolic OTCase as a reference point

Values are representative of two experiments; variations are less than $5 \%$. The titre (nl of antibody required to precipitate $75 \%$ of 10 units of $P$. aeruginosa anabolic OTCase) was $36 \mathrm{nl}$.

Enzyme source

Pseudomonas aeruginosa PAO

Pseudomonas stutzeri ATCC 11607

Pseudomonas fluorescens ATCC 13525

Pseudomonas mendocina NCIB 10541

Pseudomonas syringae ATCC 19310

Pseudomonas pseudoalcaligenes ATCC 17440

Pseudomonas putida IRC 204

Pseudomonas putida ATCC 12633

Pseudomonas aureofaciens ATCC 13985

Pseudomonas caryophilli ATCC 25418

Pseudomonas cepacia NCTC 10743

Pseudomonas acidovorans IP 60-78

Pseudomonas testosteroni ATCC 11996

Pseudomonas maltophilia ATCC 13607

Pseudomonas indigofera ATCC 19706

Methanobacterium thermoaceticum

Agrobacterium tumefaciens R10
Taxonomic classification (according to Palleroni et al., 1974)

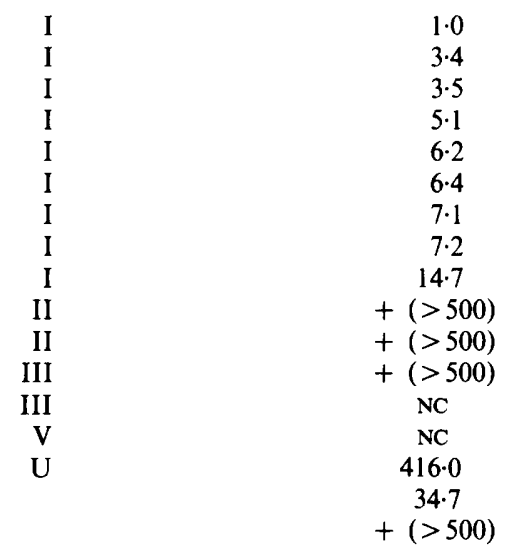

Abbreviations: NC, no cross-reaction; U, unclassified; + , positive cross-reactivity at the highest level of antiserum $(100 \mu \mathrm{l})$.

was observed, even with the highest level of antisera $(10 \mu \mathrm{l})$. These observations led to the conclusions: (i) that antibodies are directed against determinants which undergo conformational changes upon the addition of ligands; (ii) that some antibodies are able to combine with only one conformational state. Since a different quaternary structure is reached in the presence of either $\mathrm{KCl}$ or ethylene glycol, two possibilities must be considered to explain the fact that antibodies react more effectively in the presence of $\mathrm{KCl}$, which favours the nonameric structure of the enzyme: (i) some antigenic determinants are formed by the assembly of the trimeric structures into the nonameric form; (ii) some antigenic determinants have a different conformation in the nonameric and in the trimeric states.

Some cross-reactivity was observed with the $A$. formicans catabolic OTCase, which also exists as various oligomeric species. The ID value for the catabolic OTCase relative to the anabolic enzyme was 100. However, no cross-reaction could be detected, either with the anabolic OTCases from various arginine prototrophic organisms, or with catabolic OTCases from other genera used in this study.

\section{Specificity of the antiserum to P. aeruginosa anabolic OTCase}

Specific antisera gave heterologous cross-reactions of various intensities with the anabolic OTCases from Pseudomonadaceae (Table 3). The anabolic OTCases from the Pseudomonas of group I according to the classification of Palleroni et al. (1974) showed heterologous crossreactions of considerable strength with antiserum to $P$. aeruginosa anabolic OTCase (Table 3). Weak cross-reactions were observed with the anabolic enzyme from $P$ seudomonas of group II $(P$. caryophilli and $P$. cepacia). With the two strains of group III used in this study, one, $P$. acidovorans showed a weak cross-reaction while the other, $P$. testosteroni, showed no crossreactivity. $P$. maltophila, a strain of group V, did not show any reaction at all. Outside the genus Pseudomonas, only the anabolic enzyme of Agrobacterium cross-reacted with the antiserum to anabolic OTCase while, outside the eubacterial kingdom, cross-reactivity was also observed with Methanobacterium thermoautotrophicum. Neither the anabolic enzymes of Enterobacteriaceae, Aeromonas, Bacillus licheniformis and Arthrobacter, nor the catabolic OTCases from Pseudomonas, Aeromonas, Bacillus licheniformis and Streptococcus faecalis showed any crossreaction with the antiserum. 
Table 4. Indices of dissimilarity for OTCases with Streptococcus faecalis catabolic OTCase as a reference point

Values are the means of a single experiment done in duplicate. The titre ( $\mathrm{nl}$ antiserum required to precipitate $75 \%$ of 10 units of $S$. faecalis catabolic OTCase) was $41 \mathrm{nl}$.

\section{Enzyme source}

Streptococcus faecalis ATCC 11700

Streptococcus faecalis IRC

Streptococcus faecalis ATCC 14508

Streptococcus faecium ATCC 9790

Streptococcus lactis NCIB 8586

Lactobacillus brevis IP 7135

Lactobacillus fermentum ATCC 9338

Staphylococcus aureus Cowan ATCC 12595

Bacillus licheniformis ATCC 14580

Aeromonas formicans NCIB 9232

Pseudomonas putida

Pseudomonas aeruginosa PAO

Escherichia coli $\mathrm{K} 12$ argF product

Escherichia coli K12 argI product

$\begin{array}{cc}\begin{array}{c}\text { Enzyme } \\ \text { function }\end{array} & \begin{array}{c}\text { Index of } \\ \text { dissimilarity }\end{array} \\ \text { C } & 1 \cdot 0 \\ \text { C } & 1 \cdot 1 \\ \text { C } & 1 \cdot 0 \\ \text { C } & 3 \cdot 1 \\ \text { C } & 3 \cdot 4 \\ \text { C } & 2 \cdot 2 \\ \text { C } & 8 \cdot 0 \\ \text { C } & 18 \cdot 4 \\ \text { C } & 105 \cdot 0 \\ \text { C } & 178 \cdot 0 \\ \text { C } & + \\ \text { C } & \text { NC } \\ \text { A } & + \\ \text { A } & \text { NC }\end{array}$

Abbreviations: A, anabolic OTCase; C, catabolic OTCase; + , positive cross-reaction at the highest level of antiserum concentration; NC, no cross-reaction.

\section{Specificity of antiserum to PTCase}

Antibodies raised against $S$. faecalis ATCC 11700 PTCase (titre $57 \mathrm{nl}$ ) cross-reacted only with the homologous PTCase from the other $S$. faecalis species and with a PTCase found in a Lactobacillus brevis isolate. The index of dissimilarity in the case of $S$. faecalis IRC, $S$. faecalis ATCC 14508 and Lactobacillus brevis IP 7135 were $1 \cdot 4,1 \cdot 5$ and $2 \cdot 1$ respectively. No PTCase was produced by other lactic bacteria, or by any other bacterium used in this study. None of the five antisera to OTCases used in this study showed any cross-reactivity with these PTCases.

\section{Specificity of the antiserum to $S$. faecalis catabolic OTCase}

The OTCases of faecal and other streptococci, and of Lactobacillus and Staphylococcus show a high degree of resemblance to each other (Table 4). These species form a super cluster within the so-called clostridia subbranch of Gram-positive bacteria. That these organisms are distantly related to the genus Bacillus (Stackebrandt \& Teuber, 1988) is confirmed by the weaker reactivity of the Bacillus licheniformis catabolic OTCase with the antiserum to $S$. faecalis OTCase. Outside the genus, cross-reactions were also observed with the catabolic enzyme of A. formicans. At the highest level of antiserum used $(100 \mu \mathrm{l})$, an extremely weak cross-reaction was produced with the $P$. putida catabolic OTCase or $E$. coli $\arg F$ product. However, no crossreaction could be detected with the $P$. aeruginosa catabolic enzyme or with the anabolic enzymes of either Enterobacteriaceae, Pseudomonadaceae, Bacillus or Aeromonas.

$$
\mathrm{NH}_{2} \text {-terminal sequences }
$$

The alignment of the $\mathrm{NH}_{2}$-terminal sequences of several carbamoyltransferases is shown in Fig. 2. Outside bacterial genera the comparison of the OTCases from yeast, Aspergillus and man

Fig. 2. Comparison of the $\mathrm{NH}_{2}$-terminal amino acid sequences of carbamoyltransferases. The sequences are aligned on $E$. coli catalytic ATCase (pyrB product) (Hoover et al., 1983) according to their best scoring using the algorithm of Pearson \& Lipman (1988). +, Residue positively charged; residue negatively charged; $O$, hydrophobic residue, $O$, polar residue. The $\arg I$ and $\arg F$ sequences of E. coli K12 were obtained from Bencini et al. (1983) and Van Vliet et al. (1984). The ATCase sequence of $B$. subtilis is from Lerner \& Switzer (1986). The eukaryotic carbamoyltransferase sequences (Saccharomyces, Aspergillus and human) are from Huygen et al. (1987). The arcB sequences of $P$. putida and $A$. formicans are from Falmagne $e t$ al. (1985), and the $\operatorname{arc} B$ and $\arg F$ products of $P$. aeruginosa from Baur et al. (1987) and Itoh et al. (1988). The amino sequences of $S$. faecalis catabolic OTCase and PTCase and $\boldsymbol{A}$. formicans anabolic OTCase were determined in this study. 


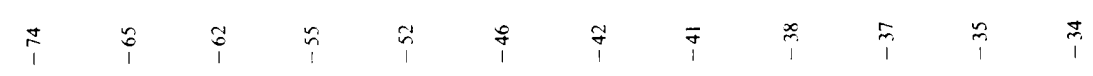

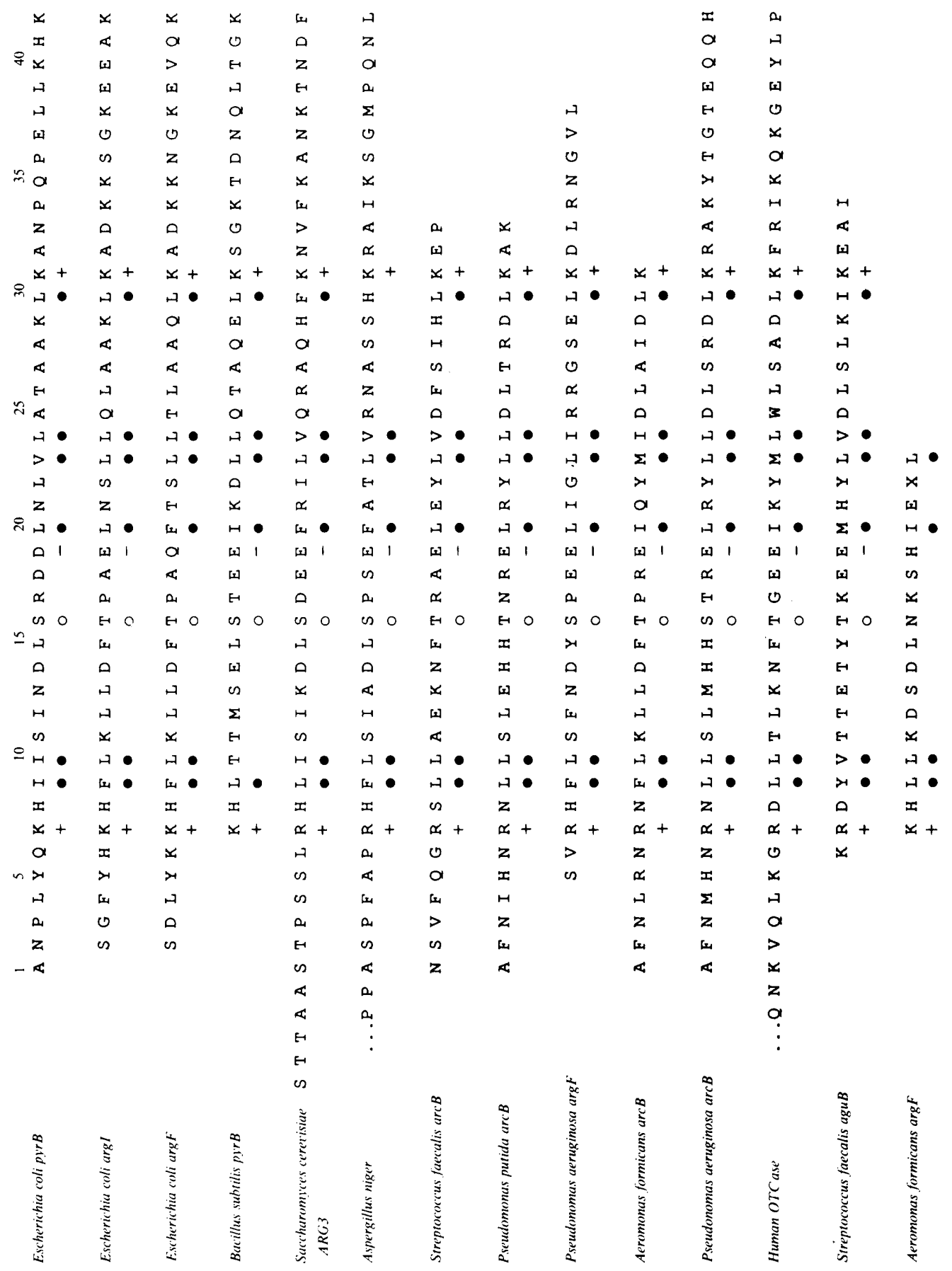



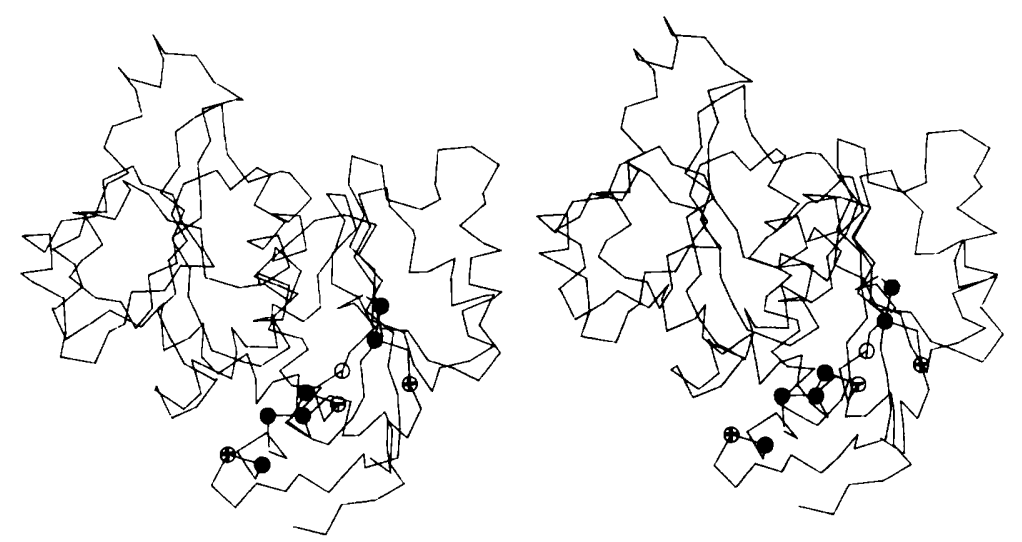

Fig. 3. Stereo view of the backbone folding observed in the crystalline state for $E$. coli catalytic ATCase. Modelling of $E$. coli ATCase was done using the coordinate of Monaco et al. (1978) and records in the protein data bank (Bernestein et al., 1977). Amino acids which appear to be conserved in the eleven sequences shown in Fig. 2 are depicted by the same symbols as in that Figure.

are included (Huygen et al., 1987). In this study, three new $\mathrm{NH}_{2}$-terminal sequences were determined, those of $S$. faecalis OTCase and PTCase and of $A$. formicans anabolic OTCase. The alignment was performed by using the optimal algorithm of Goad \& Kanehisa (1982) as improved by Pearson \& Lipman (1988). Carbamoyltransferases are aligned according to the best scoring relative to the catalytic subunit of $E$. coli K12 ATCase, for which the crystallographic structure is known at $2.6 \AA(0.26 \mathrm{~nm})$ resolution (Kim et al., 1987). The amino acids in positions $7,9,10,16,19,20,23,24,30$ and 31 are most conserved. In the three-dimensional structure of ATCase (Fig. 3), Leu20, Val23 and Leu30 define a hydrophobic face on helix 1, which is in contact with helix 5 and 9. The side-chain of the conserved polar residues Lys 7 and Lys 31 point outside the protein while Ser 16, which is located at the beginning of helix 1, might play a role in initiating the folding of this first helix. These observations suggest that the tertiary structure observed for ATCase in the $\mathrm{NH}_{2}$-terminal region may well be maintained in the spatial arrangement of the other carbamoyltransferases.

\section{DISCUSSION}

The analogies found among the reaction mechanisms, the amino acid sequences (when available), and the prediction of secondary structures of carbamoyltransferases have suggested that these enzymes belong to a system of acquisitive evolution which has occurred following duplication of a gene encoding an ancestral broad specificity carbamoyltransferase (Legrain et al., 1974, 1977; Hoover et al., 1983; Vickers, 1981; Falmagne et al., 1985; Huygen et al., 1987; Van Vliet et al., 1988).

The complete primary structures of eight carbamoyltransferases are now available: their comparison shows similarity at the amino acid sequence level between $E$. coli ATCase and anabolic as well as catabolic OTCases.

The determination of amino acid sequence and three-dimensional structure of proteins is a cumbersome task. Immunochemical methods could be a shortcut in the detection of structural similarities among proteins. The catabolic enzymes are usually larger proteins composed of six, eight, nine or more identical subunits while the enterobacterial anabolic OTCases generally occur as trimeric molecules. Nevertheless, our study confirms the strong immunological relationships of the catabolic OTCases from various genera with the anabolic enzymes of enterobacteria. Consequently, the variation in quaternary structure between the catabolic OTCases and the anabolic OTCases of enterobacteria reflects the different modes of assembly of a polypeptide whose structure has been relatively conserved.

In contrast, both catabolic OTCase and anabolic OTCase from enterobacteria showed no immunological relationships with the anabolic enzymes from either Pseudomonas, Aeromonas or Bacillus as well as OTCases from other genera. The failure to detect immunological cross- 
reactivity among related proteins does not, however, conclusively eliminate the existence of structural similarities between them. This is illustrated by the strong cross-reactivity with the $E$. coli $\arg F$ product and the weak or nonexistent cross-reactivity with the $E$. coli arg $I$ product of the antisera to catabolic OTCase of either Pseudomonas or Aeromonas. Yet these two E. coli isoenzymes bear strong sequence similarities and show an overall identity of $86 \%$ at the amino acid sequence level (Van Vliet et al., 1984).

In much the same way, the antigenicity of a protein is also critically dependent on its conformation, as is shown with $A$. formicans anabolic OTCase which exists in various oligomeric forms. The specific antiserum appears to be raised against the higher oligomeric structure and shows less affinity for the trimeric structure.

The use of $P$. aeruginosa anabolic OTCase as an antigen was also justified because this enzyme showed kinetic properties which confer unidirectionality toward citrulline synthesis, by contrast with other catabolic or anabolic OTCases which can work in vitro and in vivo in both directions of the reaction (Legrain et al., 1972, 1977). The determination of the sequence of this enzyme (Itoh et al., 1988) showed only $45 \%$ identity with $E$. coli OTCase isoenzymes or with $P$. aeruginosa catabolic OTCase. No cross-reaction could be observed between the $P$. aeruginosa anabolic OTCase and antisera raised against the $P$. aeruginosa catabolic OTCase and the homologous enzymes of the same or other genera, nor with the antisera raised against the $E$. coli (Falmagne $e t$ al., 1985) and $A$. formicans anabolic OTCases.

Conversely, no cross-reaction was observed between the antiserum to $P$. aeruginosa anabolic OTCase and catabolic OTCase as well as anabolic OTCases from the enterobacteria. Strong positive cross-reactions were, nevertheless, observed with antisera to $P$. aeruginosa anabolic OTCase and anabolic OTCase from Pseudomonas strains of group I as defined by Palleroni et al. (1974). Positive cross-reactions were also obtained with Pseudomonas strains of groups II and IV; these groups represent, in fact, entirely separate rRNA homology groups, only interrelated at supragenic or superfamilial level (Byng et al., 1983). Outside the pseudomonads, positive cross-reaction was observed with the enzyme of Agrobacterium and, outside the eubacterial line, with an Archaeobacterium, indicating that structural similarities may be conserved even between $P$. aeruginosa anabolic OTCase and members outside the eubacterial line.

$\mathrm{X}$-ray analysis of $P$. aeruginosa catabolic OTCase is now in progress. Comparison with the results obtained by $\mathrm{L}$. Kuo with the $E$. coli anabolic OTCase will be essential in understanding the molecular components which determine the behaviour of these immunologically related enzymes as well as the allosteric properties of the $P$. aeruginosa catabolic enzyme. On the other hand, as numerous amino acid sequence segments are highly conserved in many parts of these OTCases whose primary structure are known, model-building using computer graphics could be used with a high degree of confidence to quickly derive appropriate models for a number of other carbamoyltransferases from several origins. This work is now under progress in our laboratories.

We thank Françoise Huet, Annick Mercenier, Christiane Legrain, Jean-Paul Simon and Brigitte Wargnies for their participation in some of the experiments, Jean-Marc Godart for his technical assistance and Professor A. Piérard for reading and correcting the manuscript.

This work is supported by grants 2.4521.83 and 2.9003.88 from the Belgian Fund for Joint Basic Research, by a Research Contract EEC no. BAP-0345-B (GDF) and by an 'Action de Recherche Concertée' financed by the Belgian Government. J.-L.D. and V.S. are Research Associates of the National Fund for Scientific Research (Belgium).

\section{REFERENCES}

Baur, H., Stalon, V., Falmagne, P., Lüthi, E. \& HAAS, D. (1987). Primary and quaternary structure of the catabolic ornithine carbamoyltransferase from Pseudomonas aeruginosa. Extensive sequence homology with the anabolic ornithine carbamoyltransferases of Escherichia coli. European Journal of Biochemistry 166, 111-117.

Bencini, D. A., Houghton, J. E., Hoover, T. A., FoltermanN, K. F., Wild, J. R. \& O'Donovan,
G. A. (1983). The DNA sequence of $\arg I$ from $E$. coli K-12. Nucleic Acids Research 11, 8509-8518.

Bernestein, F. C., Koetzle, T. F., Williams, G. J. B., MEYeR, E. F., JR, BRICE, M. D., RoDGERS, J. R., Kennard, O., Shimanouchi, T. \& Tasumi, M. (1977). The protein data bank: a computer-based archival file for macromolecular structures. Journal of Biology 112, 535-542.

Broman, K., Lauwers, N. \& Stalon, V. (1978). 
Oxygen and nitrate in the utilization by Bacillus licheniformis of the arginase and arginine deiminase routes of arginine catabolism, and other factors affecting their synthesis. Journal of Bacteriology 135, 920-927.

Byng, G. S., Johnson, J. L., Whitaker, R. J., Gherna, R. \& Jensen, R. A. (1983). The evolutionary pattern of aromatic amino acid biosynthesis and the emerging phylogeny of pseudomonad bacteria. Journal of Molecular Evolution 19, 272-282.

Cunin, R., Glansdorf, N., Piérard, A. \& Stalon, V. (1986). Biosynthesis and metabolism of arginine in bacteria. Microbiological Reviews 50, 314-352.

Falmagne, P., Portetelle, D. \& Stalon, V. (1985). Immunological and structural relatedness of catabolic ornithine carbamoyltransferases and the anabolic enzymes of enterobacteria. Journal of Bacteriology 161, 714-719.

Goad, W. B. \& Kanehisa, M. L. (1982). Pattern recognition in nucleic acid sequences. A general method for finding local homologies and symmetries. Nucleic Acids Research 10, 247-263.

Halleux, P., Legrain, C., Stalon, V., Piérard, A. \& WiAme, J. M. (1972). Regulation of the catabolic ornithine carbamoyltransferase of Pseudomonas fluorescens. A study of the quaternary structure. European Journal of Biochemistry 31, 386-393.

Hoover, T. A., Roof, W. D., Foltermann, K. F., O'Donovan, G. A., Bencini, D. A. \& Wild, J. R. (1983). Nucleotide sequence of the structural gene (pyrB) that encodes the catalytic polypeptide of aspartate carbamoyltransferase of Escherichia coli. Proceedings of the National Academy of Sciences of the United States of America 80, 2462-2466.

HoPP, T. P. \& WooDs, R. (1981). Prediction of protein antigenic determinants from amino acid sequences. Proceedings of the National Academy of Sciences of the United States of America 78, 3824-3828.

Huygen, R., Crabeel, M. \& GlansdorfF, N. (1987). Nucleotide sequence of the ARG3 gene of the yeast Saccharomyces cerevisiae encoding ornithine carbamoyltransferase. European Journal of Biochemistry 166, 371-377.

Itoh, Y., Soldati, L., Stalon, V., Falmagne, P., Terawaki, Y., Leisinger, T. \& HaAs, D. (1988). Anabolic ornithine carbamoyltransferase of Pseudomonas aeruginosa: nucleotide sequence and transcriptional control of the $\arg F$ structural gene. Journal of Bacteriology 170, 2725-2734.

KIM, K. H., Pan, Z., HonzatKo, R. B., Ke, H. M. \& LIPSCOMB, W. N. (1987). Structural asymmetry in the CTP-liganded form of aspartate carbamoyltransferase from Escherichia coli. Journal of Molecular Biology 196, 853-875.

Legrain, C. \& Stalon, V. (1976). Ornithine carbamoyltransferase from Escherichia coli W. Purification, structure, and steady-state kinetic analysis. European Journal of Biochemistry 63, 289-301.

Legrain, C., Halleux, P., Stalon, V. \& GlansDORFF, N. (1972). The dual genetic control of ornithine carbamoyltransferase in Escherichia coli: a case of bacterial hybrid enzymes. European Journal of Biochemistry 27, 93-102.

Legrain, C., Stalon, V., Noullez, J. P., Mercenier, A., Simon, J. P., Broman, K. \& Wiame, J. M. (1977).
Structure and function of ornithine carbamoyltransferases. European Journal of Biochemistry 80, 401409.

Lerner, C. G. \& Switzer, R. L. (1986). Cloning and structure of the Bacillus subtilis aspartate transcarbamoylase gene. Journal of Biological Chemistry 261, $11156-11165$.

Marshall, M. \& Cohen, P. P. (1972). Ornithine transcarbamylase from Streptococcus faecalis and bovine liver: isolation and subunit structure. Journal of Biological Chemistry 247, 1641-1653.

MeILE, L. \& LeISINGER, T. (1984). Enzymes of arginine hiosynthesis in methanogenic bacteria. Experientia 40, 899-890.

Monaco, H. L., Crawford, J. L. \& Lipscomb, W. N. (1978). Three-dimensional structures of aspartate carbamoyltransferase from Escherichia coli and its complex with cytidine triphosphate. Proceedings of the National Academy of Sciences of the United States of America 75, 5276-5280.

Palleroni, N. J., Kunisawa, R., Contopoulou, R. \& DOUDOROFF, M. (1974). Nucleic acid homologies in the genus Pseudomonas. International Journal of Systematic Bacteriology 23, 333-339.

Pearson, W. R. \& Lipman, D. J. (1988). Improved tools for biological sequences comparison. Proceedings of the National Academy of Sciences of the United States of America 85, 2444-2448.

Stalon, V. \& MERCEnIER, A. (1984). L-Arginine utilization by Pseudomonas species. Journal of General Microbiology 130, 69-76.

Stalon, V., Ramos, F., Piérard, A. \& Wiame, J. M. (1972). Regulation of the catabolic ornithine carbamoyltransferase of Pseudomonas fluorescens: a comparison with the anabolic transferase and with a mutationally modified catabolic transferase. European Journal of Biochemistry 29, 25-35.

StackebrandT, E. \& Teuber, M. (1988). Molecular taxonomy and phylogenetic position of lactic acid bacteria. Biochimie 70, 317-324.

Van Vliet, F., Cunin, R., Jacobs, A., Piette, J., Gigot, D., Lauwereys, M., Piérard, A. \& GlansDORFF, N. (1984). Evolutionary divergence of genes for ornithine and aspartate carbamoyltransferases complete sequence and mode of regulation of the Escherichia coli argF gene; comparison of $\arg F$ with $\operatorname{argI}$ and pyrB. Nucleic Acids Research 12, 62776289.

Van Vliet, F., Boyen, A. \& Glansdorff, N. (1988). On interspecies gene transfer: the case of the $\arg F$ gene of Escherichia coli. Annales de l'Institut Pasteur/ Microbiologie 139, 493-496.

VICKERS, L. P. (1981). Trimeric enzymes in carbamoylphosphate metabolism. Trends in Biochemical Sciences 6, 11-12.

Wargnies, B., Lauwers, N. \& Stalon, V. (1979). Structure and properties of the putrescine carbamoyltransferase of Streptococcus faecalis. European Journal of Biochemistry 101, 145-152.

Westhof, E., Altshuh, D., Moras, D., Bloomer, A. C., Mondragon, A., Klug, A. \& Van RegenMORTEL, M. H. V. (1984). Correlation between segmental mobility and the location of antigenic determinants in proteins. Nature, London 311, 123 134. 